\title{
La muerte y los niños
}

\author{
CRISTIÁN ZAÑARTU S. ${ }^{1}$, CHRISTIANE KRÄMER K. ${ }^{2}$, MARÍA ANGÉLICA WIETSTRUCK P. ${ }^{3}$ \\ 1. Interno de Medicina. Pontificia Universidad Católica de Chile. \\ 2. Psicóloga . \\ 3. Hemato-Oncólogo Pediatra, Departamento de Pediatría, Pontificia Universidad Católica de Chile.
}

\begin{abstract}
\section{Children and Death}

The present article intends to create a more healthy relationship between death and the pediatric patient and, besides, deliver minimal tools to physicians, parents and children, in order to live this experience in a better way. To achieve this purpose, we based our study on bibliographical research and patientspsychologists-physicians experiences from pediatric oncology at our hospital. Piaget defines a clear progression in the child idea of death, ranging from intuition to abstraction. Although language will not be the same in every case, there is no minimum age for a child to deal in its own way with the death of the people who surround him. Not allowing children to suffer for their loved persons will not help them heal the wounds they experiment. An abnormal approach can determine mistaken, painful, and guilty conclusions that we must avoid.
\end{abstract}

(Key words: Death, pediatrics, mortality, ilness).

Rev Chil Pediatr 2008; 79 (4): 393-397

\section{RESUMEN}

El tema de la muerte en los niños está pobremente integrado como una realidad en la práctica clínica. El presente artículo pretende lograr un acercamiento más sano a aquel escenario que reúne a la muerte y al paciente pediátrico, y entregar las mínimas herramientas a médicos, padres y a los mismos niños, para vivir estas experiencias de mejor manera. Para lograrlo, se basa en revisión bibliográfica, y experiencias de pacientes, médicos y psicólogos del departamento de oncología pediátrica del Hospital Clínico de la Universidad Católica. La idea de muerte en el niño sigue una cadena evolutiva, desde la intuición hasta la abstracción. A pesar de que el lenguaje no será el mismo en todos los casos, no hay edad ideal para permitir a un niño lidiar con la muerte de quienes lo rodean y la suya propia. Ciertos errores clásicos, algunos amparados en una buena intención, pueden generar efectos adversos. El no hacer parte a los niños del duelo de sus queridos, no les permite cerrar las heridas que experimentan en su manera. Duelos anormales pueden llevarlo a conclusiones erradas, dolorosas, y culpógenas, que se deberían evitar.

(Palabras clave: Muerte, pediatría, mortalidad, duelo, enfermedad).

Rev Chil Pediatr 2008; 79 (4): 393-397

Trabajo recibido el 09 de noviembre de 2007, devuelto para corregir el 03 de enero de 2008, segunda versión el 11 de abril de 2008, aceptado para publicación el 16 de mayo de 2008.

Correspondencia a:

Cristián Zañartu S.

E-mail: cristianzanartu@gmail.com 


\section{Introducción}

El tema de la muerte en los niños está pobremente integrado hoy como una realidad en la práctica clínica. La estadística es escasa en cuanto al manejo psicológico y a las consecuencias de este manejo en el niño con una enfermedad terminal y en sus hermanos. Por otro lado, se reporta un doble en la tasa de suicidios, sobre todo en el primer mes, de padres que han perdido un hijo con enfermedad, con respecto a cohortes de padres con hijos sanos $^{1}$. La magnitud de esta asociación queda demostrada en recientes reportes de la literatura, donde padres han ocupado los remanentes de medicamentos paliativos del hijo difunto para infringirse daño ${ }^{2}$. No hay estudios de este tipo en niños. Sí se ha descrito la clara asociación de muertes parentales o de hermanos en la infancia, y psicopatología en la adultez, sobre todo cuando hay duelos anormales ${ }^{3}$. Claramente estamos describiendo un escenario muy complejo, para argumentar la necesidad de detenerse ante la psiquis del propio niño enfermo, o la del hermano en duelo. Estudios basados en entrevistas póstumas a padres, hijos y hermanos son claros en concluir que en estas circunstancias, la comunicación con el profesional de la salud es generalmente insuficiente en cantidad y calidad, y se ha fallado en reconocer la necesidad de apoyo psicológico o psiquiátrico en los niños ${ }^{4}$.

Se presenta la siguiente revisión en el objeto de profundizar en 2 aspectos del tema, el primero, cómo se enfrenta un niño a la idea de su propia muerte, cuando es llevado a circunstancias que la hacen potencial o inminente, y luego, cómo ve y recibe un niño la muerte de un ser querido.

A partir de la revisión bibliográfica y de experiencias con pacientes, médicos y psicólogos del programa de oncología pediátrica de la Pontificia Universidad Católica, pretendemos entregar algunas recomendaciones de cómo abordar, en lo posible, el tema de la muerte con los niños.

\section{Pacientes y Métodos}

La búsqueda bibliográfica se realizó en
Pubmed conjugando los términos MESH de muerte (death) con niños (children), y de duelo (bereavement) con niños. Los resultados fueron seleccionados dando preferencia a artículos de revisión y aquellos abocados al manejo de información y variables psicológicas con los padres y niños, seleccionando finalmente 12 estudios. Se hizo revisión de capítulos de escritos de la recientemente difunta Dra. Kubler Ross (QEPD), pionera en estudios de muerte y niños, y de J. W. Worden, autor clave en la temática del duelo. A su vez, se obtuvo información de la experiencia tanto por parte del equipo médico como del psicológico adyuvante, a lo largo de los años 2005 a 2007 con familiares y pacientes del servicio de oncología pediátrica del Hospital Clínico de la Universidad Católica de Chile.

\section{Desarrollo}

\section{Idea de muerte}

La comprensión de la muerte va cambiando con la edad ${ }^{5}$. Antes de los dos años existe la sensación de ausencia y presencia, asociando a ello manifestaciones de angustia, no hay pensamiento operacional ni la capacidad de integrar un concepto como la muerte. Según Piaget ${ }^{6}$, los niños a esta edad se caracterizan por un desarrollo sensorio-motor, basados más que nada en los reflejos, y en las conductas que van adquiriendo con la experiencia, describiéndose que ante el dolor, se pueden volver apáticos y presentar alteraciones somáticas ${ }^{7}$.

Entre los tres y los siete años, el pensamiento pre-operacional, centrado en la intuición, trae consigo la búsqueda de mecanismos causaefecto para lo que va sucediendo. Es ahora que tiene cabida la idea de la muerte, pero como un fenómeno reversible o temporal, con atribuciones mágicas que responden a su pensamiento pre-operacional, y el concepto puede adquirir propiedades tenebrosas. Entre los siete y doce años, aparece el pensamiento operacional, donde se van adquiriendo los diferentes elementos que Piaget define como conformadores de una idea de muerte apropiada (inmovilidad, universalidad, irrevocabilidad, entre otros). Por otro lado, el niño es capaz de ver las situaciones bajo 
distintos puntos de vista, pero aún es incapaz de generar un pensamiento abstracto para comprender lo permanente y absoluto de la muerte.

Es desde los doce años de edad que se presenta el concepto de muerte aliado a la capacidad de abstracción. Ahora, el niño sí se empieza a acercar al pensamiento adulto y generar sus ideas propias, avanzando del pensamiento concreto al hipotético-deductivo, lo que quizá puede hacerlo pensar en la muerte como concepto universal y llenarse de preguntas ¿Si él murió, moriremos todos? ¿Si me enfermo también puedo morir?, etc.

Es de hacer notar que estos rangos de edad pueden ser variables, dependiendo de la experiencia y madurez de cada niño.

\section{Los riesgos de una "buena intención"}

Los niños van adquiriendo herramientas a medida que se desarrollan. Sin embargo, la carencia de ellas no justifica mantenerlos aislados de la verdad ${ }^{8}$. Existe una fuerte tendencia protectora a ocultar los diagnósticos ominosos al niño enfermo, a negar la muerte próxima y, con igual afán, a no hacerlos parte del duelo por un hermano u otro familiar, muchas veces porque los adultos se sienten poco preparados para dar esa información. Y no es fácil: un duelo anormal puede causar estragos en el desarrollo emocional hacia la adultez ${ }^{9}$.

Por ello, lo primero es plantearse el nivel de desarrollo del niño. Si tiene menos de dos años, la comunicación no radica en las palabras: el soporte físico, la cercanía de sus padres y el tratamiento del dolor son los elementos claves. Posteriormente, el desarrollo del pensamiento pre-operacional y operacional, trae consigo la capacidad de entender el concepto de la muerte, pero también el riesgo de violentas asociaciones inapropiadas que se desprenden de actitudes y frases de los adultos, de las que hablaremos a continuación.

\section{Hablar con los niños}

Habrá tres conceptos principales para tener en mente al conversar con los niños sobre la muerte de un hermano, de otro ser querido, o la de ellos mismos ${ }^{10}$. Para cada uno, señalaremos errores que se pueden evitar:
1) Entregar un concepto claro de muerte. La primera fase de un duelo es reconocer la realidad de muerte, para lo cual se debe tener en cuenta que no se va a ver nuevamente al difunto. Para niños en fase preoperacional y operacional una buena definición sería: "es no volver a vivir, es no respirar, no sentir dolor, no moverse, no hablar ni tener hambre"11. La doctora Kübler-Ross ${ }^{12}$, se refiere a la mente de los niños como un escenario más sano que el de los adultos, para trabajar en paz y amorosamente el tema de la muerte, aún el de la propia. El reto está en ocupar las palabras apropiadas, y no entregarles nuestros propios miedos.

Esconderles el conocimiento de su propia enfermedad ("conspiración del silencio") es un error común. No sólo los adultos ${ }^{13}$, sino que los niños también sospechan sus diagnósticos con relativa claridad, y los viven con miedo en soledad, jugando al juego de los padres. Se pierde la posibilidad de decirse que se quiere, afrontar los miedos, de llorar y aceptar juntos ${ }^{9}$. Nuestra misión es hacerles entender a los padres, en un proceso que puede tomar su tiempo, la importancia de decirles la verdad a los niños en relación al diagnóstico, con todos sus nombres, aclarándoles las dudas y temores que puedan surgir, y que se den tiempo de conversarlo y llorar con sus padres.

El tema es más difícil cuando se trata de abordar la propia muerte. Aún en los adultos que manifiestan su deseo de saber si van a morir, la certeza de la muerte inminente crea angustias que a veces superan la racionalidad. En el niño, esta necesidad es mucho menos perentoria: no hay deudas que saldar, no hay trabajos que concretar; más bien, mantener la esperanza en el niño puede ser de ayuda en el proceso que se viene por delante. Y si preguna ¿es cierto que me voy a morir?, la respuesta podría ser: "te estamos ayudando para que eso no suceda".

Por otra parte, el no hacerlos partícipes de la enfermedad o muerte de sus hermanos u otros seres queridos, no llevarlos al funeral, no compartir con ellos la pena por la pérdida, podría traer como resultado la dificultad de iniciar y elaborar el duelo.

La primera fase de un duelo, según Worden ${ }^{14}$, es aceptar la realidad de la pérdida. En este 
sentido, el funeral puede ser una instancia facilitadora para empezar a vivir la pena, tanto niños como adultos. Al consolar con argumentos que confunden: "Vamos a ver de nuevo a tu hermano", el niño con pensamiento preoperacional $\mathrm{u}$ operacional no abstracto, no tendrá claridad de lo que pasó con su hermano. Se retrasa el inicio del duelo y su desarrollo se entorpece ${ }^{6}$.

2) Generar un sentimiento de protección en el niño ante la pérdida de un ser querido. La etapa final del duelo contempla que se encuentre un lugar emocional privado para el difunto, que permita amar con libertad a los otros ${ }^{13}$. Independientemente de este paso en los padres, para el hermano este proceso puede ser muy confuso y doloroso en la soledad ya citada. En el caso de la muerte de un hijo será normal, como una fase inicial, que los padres intenten no desprenderse del difunto buscándolo en un nuevo hijo. El hijo sobreviviente pensará: "No fui suficiente como reemplazo". Es importante dejar claro que nadie remplaza a otra persona, ni un padre a una madre, ni un hijo a otro hijo.

A pesar de que son muchas veces usadas con buena intención, frases como: "Todos vamos a morir algún día" pueden asustar y generar ideas como: "¿Mis papás morirán igual?" "¿Me voy a morir?" El niño se siente desprotegido y se asusta. Recordar percatarse de cómo se aproxima el niño a la muerte, de acuerdo a la etapa por la que esté pasando 6 .

3) Prevenir o contener, cuando ya estén presentes, sentimientos de culpa y rabia. "Idolatrar" al hermano muerto cuando se ha muerto un hijo. Multiplicar sus fotos en la casa, idealizar su persona. Todas estas actitudes tendrán un efecto negativo en los hermanos que sobreviven, ya que en la natural competencia por destacar ante los padres, el hermano vivo sentirá envidia y rabia hacia el hermano muerto. Estos sentimientos se cargan en soledad, sin contención y con gran culpa ${ }^{10}$.

"Dios se lo llevó, porque era bueno" - es una frase bien intencionada común en padres en duelo. La reacción nuevamente puede no ser la esperada: "¿Por qué yo me quedo vivo?, ¿Será porque soy malo?", "Dios se lleva a las personas intempestivamente". Es dificil enmarcar esto en la imagen de un Dios amoroso. Previo a la capacidad de abstracción, no se tolera ni entiende un Dios ambivalente.

Descubrimos que no es poco frecuente que los hermanos pequeños se hayan dicho entre ellos frases violentas durante discusiones o peleas: "Ojalá te mueras". Esto puede generar un sentimiento de culpa, cuya vergüenza e intensidad criminal, hace que lo experimenten y resuelvan por sí solos y muchas veces parcialmente.

Un fenómeno bastante común, es la incapacidad de los padres para contener a los hermanos al momento de perder a un hijo. Se encierran, comprensiblemente, en la supervivencia de su propia crisis ${ }^{1,2}$. Pero es en este proceso, en que aquel que perdió a un hermano, pierde también a sus padres. El hijo contiene al padre, en la medida que ocultar su propia pena lo protege. Estudios observacionales han mostrado por ejemplo, que la mayoría de los hermanos que sobreviven, cooperan más con las funciones de la casa ${ }^{15}$. Es la única forma en la que el padre puede volver a ocupar su verdadero rol, y el niño aumenta su propia posibilidad de subsistir. Así, paradojalmente, el hijo carga con el padre, en la esperanza de volver a la normalidad.

\section{Conclusión}

Se han descrito las etapas del desarrollo del pensamiento aliados a la idea de muerte, las principales tareas comunicativas a cumplir, y errores comunes de padres y profesionales del área de la salud al hablar o no hablar con los niños. Al no discutir abiertamente la muerte, no se contendrán los miedos de los hijos. Si los padres ocultan sus sentimientos, el niño percibirá que no se le está diciendo la verdad. Si no se explica qué es la muerte, no se puede iniciar el duelo. Así, se debe instar a los padres a hacer un esfuerzo, en la mitad de su propio dolor, para contener a los pequeños, dejar prevalecer la transparencia y no lanzar frases fáciles con resultados posiblemente negativos.

Dar una idea completa del manejo de situaciones de este calibre en esta revisión, es una ambición exagerada. Sin embargo, en el dificul- 
toso escenario descrito, con padres acongojados y un cuerpo de profesionales de la salud que no siempre sabe qué rol jugar, intentamos transmitir un mensaje de alerta, que motive un cuidado y cercanía responsables en el tema de la muerte con los niños. Aceptar la muerte como algo natural en nuestra cultura es difícil, más aún en el caso de los niños. Esperamos que este texto sea una herramienta de apoyo para enfrentarnos al paso de la vida a la muerte y ayudar como médicos y pediatras, a la familia en este proceso, intentando también a través de los ojos de los niños, ver a la muerte con mayor naturalidad y esperanza.

\section{Referencias}

1.- Vrabtchev $S V$ : Grieving the death of a child. Supporting health professionals who care for grieving patients may benefit all. BMJ 2006; 332: 794-5.

2.- Davies DE: Parental suicide after the expected death of a child at home. BMJ 2006; 332: 647-8.

3.- Christ GH, Christ AE: Current Approaches to Helping Children Cope with a Parent's Terminal Illness. CA Cancer J Clin 2006; 56: 197-212.

4.- Meert KL, Eggly S, Pollack M, et al: Grieving the death of a child. Supporting health professionals who care for grieving patients may benefit all. BMJ 2006; 332: 794-5

5.- Davies DE: Parental suicide after the expected death of a child at home. BMJ 2006; 332: 647-8.

6.- Christ GH, Christ AE: Current Approaches to Helping
Children Cope with a Parent's Terminal Illness. CA Cancer J Clin 2006; 56: 197-212.

7.- Meert KL, Eggly S, Pollack M, Newth JL, Dean JM, Willson DF, Nicholson C and The National Institute of Child Health and Human Development Collaborative Pediatric Critical Care Research Network: Parents' Perspectives Regarding a Physician-Parent Conference after Their Child's Death in the Pediatric Intensive Care Unit. J Pediatr 2007; 151 (1): 50-55.e2.

8.- Kranzler EM, Shaffer D, Wasserman G, Davies M: Early childhood bereavement. J Am Acad Child Adolesc Psychiatry 1990; 29 (4): 513-20.

9.- Piaget $J$ : El lenguaje y el pensamiento en el niño. Barcelona, Ed. Paidos: 1967, p. 50-93.

10.- Wolfe J, Grier HE, Klar N, et al: Symptoms and Suffering at the End of Life in Children with Cancer. N Engl J Med. 2000; 342:326-333

11.- Christ GH, Christ AE: Current Approaches to Helping Children Cope with a Parent's Terminal Illness. CA Cancer J Clin 2006; 56: 197-212.

12.- Dowdney L: Childhood bereavement following parental death. J Child Psychol Psychiatry 2000; 41 (7): 81930 .

13.- Kübler-Ross E: On Children and Death. NY, USA. Ed. Scribner: 1997, 1-10, p. 196-205.

14.- Kübler-Ross E: On Children and Death". NY, USA. Ed. Scribner 1997; 126-54.

15.- Newman L: Elisabeth Kübler-Ross. BMJ 2004; 329; 627-8.

16.- Barbero $J$ : El derecho del paciente a la información: el arte de comunicar. An Sist Sanit Navar 2006; 29 (Suppl 3): 19-27.

17.- Worden JW: El tratamiento del duelo. Barcelona, Ed. Paidos Iberica: 2000, p. 27-58.

18.- Van Eerdewegh MM, MD Bieri, Parrilla RH, Clayton $P J$ : The bereaved child. Br J Psychiatry 1982; 140: 23-9. 